\title{
Influência do Strain e da Interação Spin-Órbita em um Ponto Quântico Cilíndrico
}

\section{Influence of Strain and Spin-Orbit Interaction in a Cylindrical Quantum Point}

\author{
Silvio José Prado ${ }^{1}$
}

\begin{abstract}
Resumo
Neste trabalho estudo como o strain e a interação spin-órbita Rashba afetam os níveis de energia da banda de condução em um ponto quântico cilíndrico submetido a um campo magnético externo, usando o método k•p 2x2. Os resultados apresentados mostram que a interação spin-órbita influencia fortemente os níveis de energia levantando a degenerescência mesmo em campo magnético nulo, enquanto que o strain comprime os níveis aumentando os efeitos da interação Rashba. Neste contexto, a absorção óptica intrabanda vem a ser uma ferramenta útil para se estudar os efeitos da interação spin-órbita e do strain sobre os níveis de energia dos elétrons.
\end{abstract}

Paravras chave: Interação spin-órbita tipo Rashba. Strain. Pontos quânticos. Banda de condução. Método k•p.

\begin{abstract}
In this work study how the strain and spin-orbit Rashba interaction type affect the energy levels of the conduction band in a cylindrical quantum dot, using the $2 \times 2$ method k•p. The results show that the spinorbit interaction strongly influences the energy levels of lifting the degeneracy even in zero magnetic field, while the strain compressing the levels enhance the Rashba interaction effects. In this context, the intraband optic absorption becomes a useful tool to study the effects of spin-orbit interaction and the strain on the electron energy levels.
\end{abstract}

Keywords: Spin-orbit Rashba interaction. Strain. Quantum dots. Conduction Band. k•p method

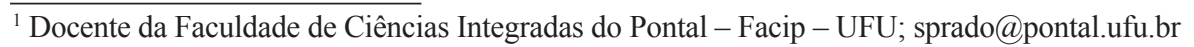




\section{Introdução}

A spintrônica é um campo da eletrônica onde tanto o spin quanto a carga do elétron são utilizados como elementos ativos nos dispositivos eletrônicos e no desenvolvimento de dispositivos de processamento quântico de informação. Desta forma controlar a dinâmica de estados quânticos coerentes chamados de qubits, é um passo fundamental em direção ao desenvolvimento dos computadores quânticos. Nesse contexto o spin dos elétrons e buracos em nanoestruturas semicondutoras é considerado como um ótimo candidato para viabilizar os qubits, pois a quantização do movimento dos portadores inibe a relaxação do spin e dos processos de decoerência, tornando os estados de spin coerentes em uma escala de tempo maior que nanosegundos (RAICEVIC; TADIC, 2009; LI; LI, 2008; PIETILÄINEN; CHAKRABORTY, 2006). $\mathrm{O}$ controle externo da dinâmica de spins pode ser feito através das propriedades eletrônicas e ópticas das nanoestruturas, sendo esta uma etapa de grande importância na execução de operações com os qubits.

Uma das técnicas de manipulação do spin tem como base a interação spin-órbita (ISO) Rashba, devido ao confinamento e a falta de simetria de inversão em nanoestruturas, criando um campo elétrico local perpendicular ao plano do elétron (MANSELYAN; CHAKRABORTY, 2009). Outra técnica de manipulação do spin é através do acoplamento spin-órbita induzido por strain na ausência de campo magnético (KATO et al., 2004). A influência do strain sobre a estrutura eletrônica é dupla. Primeiro, há uma mudança de simetria do cristal que leva à divisão de diferentes estados de spin dos elétrons, os quais são separados em spinup e spin-down, como resultado do confinamento quântico. $\mathrm{O}$ segundo efeito sobre a estrutura electrônica vem da interação spin-órbita induzida por strain, chamada de engenharia de strain, para manipulção de spin em estado sólido. Este tipo de manipulação tem sido usado em experimentos de controle de spin eletrônico, tais como na precessão e coerência de spin, propriedades de transporte entre outras (NADJ-PERGE et al., 2010; JHA et al., 2014).

Neste trabalho estudo teoricamente os efeitos da ISO tipo Rashba e do strain sobre os elétrons na banda de condução de um ponto quântico (PQ) cilíndrico de InAs crescido em um substrato de GaAs, sobre a influência de um campo magnético externo aplicado na direção-z.

\section{Teoria}

Os elétrons da banda de condução do PQ estão confinados na direção- $z$ por um potencial retangular infinito de largura $2 \mathrm{~Hz}$, enquanto que no plano- $x y$ o potencial de confinamento é do tipo parabólico, com energia $\hbar \omega_{0}\left(\mathrm{E}_{0}\right)$. Nestas condições o Hamiltoniano para um elétron com ISO Rashba e strain (RAICEVIC; TADIC, 2009) é dado por:

$$
\begin{aligned}
H & =\frac{1}{2 m^{*}} \vec{P}^{2}+\frac{1}{2} m^{*} \omega_{0}^{2} \rho^{2}+\frac{\alpha}{\hbar}[\vec{\sigma} \times \vec{P}]_{z}+\frac{1}{2} g \mu_{B} B \sigma_{z} \\
& +V_{\text {hidro }} .
\end{aligned}
$$

Nesta equação $\vec{P}$ é o operador que leva em consideração os efeitos do campo magnético, $\vec{P}=\vec{p}-\frac{e}{c} \vec{A}$, onde $\vec{p}$ é o operador sem campo e $\vec{A}$ é o vetor potencial no gauge simétrico, $\vec{A}=\frac{B}{2}(-y, x, 0)$, que corresponde a um campo magnético externo aplicado perpendicularmente ao plano de movimento dos elétrons e $\vec{\sigma}$ é o vetor das matrizes de Pauli.

O primeiro termo do Hamiltoniano corresponde à energia cinética, o segundo ao potencial de confinamento parabólico, o terceiro à ISO Rashba, sendo $\alpha$ o parâmetro que determina a intensidade da interação. O quarto termo é o acoplamento Zeeman, onde $g$ é o fator de Landé 
e $\sigma_{z}$ é projeção do spin do elétron no eixo- $z$ ( $\left.\sigma_{z}=1 / 2,-1 / 2\right)$. Finalmente, $V_{\text {hidro }}$ é o potencial efetivo devido ao strain hidrostático, que tem como causa o não casamento entre os parâmetro de rede entre InAs e GaAs.

O Hamiltoniano (1) pode ser escrito na forma matricial,

$$
H=\left(\begin{array}{ll}
H_{11} & H_{12} \\
H_{21} & H_{22}
\end{array}\right) .
$$

Os elementos $H_{11}, H_{12}, H_{21}$ e $H_{22}$ são escritos da seguinte forma:

$$
\begin{aligned}
& H_{11}=H_{22}=\frac{-\hbar^{2}}{m^{*}} \hat{P}^{2}+V_{\text {hidro }}, \\
& H_{12}=\alpha+\frac{C_{3}}{2} \varepsilon_{x y} \hat{P}_{+} \\
& H_{21}=H_{12}^{\dagger}
\end{aligned}
$$

onde os operadores $\hat{P}_{ \pm}$e $\hat{P}^{2}$ são dados por $\hat{P}_{ \pm}=\left(\frac{\partial}{\partial x} \pm i \frac{\partial}{\partial y}\right)$ e $\hat{P}^{2}=\nabla^{2}$. O strain aparece em todos os elementos do Hamiltoniano (2), representado pelo tensor $\varepsilon_{x y}$. Nos termos fora da diagonal tem-se a combinação do strain com a ISO Rashba, onde $\alpha$ determina a intensidade da interação. $O$ termo $C_{3}$ é uma constante que depende do material, $\varepsilon_{x y}$ que aparece no elemento de matriz $\mathrm{H}_{12}$ é dado por $\varepsilon_{x y}=\varepsilon_{y x}=-\left[\frac{C_{11}+2 C_{12}}{C_{11}+C_{12}+C_{44}}\right] \varepsilon_{15}$, onde $\varepsilon_{\square}$ representa o strain biaxial no plano- $x y$, com $\varepsilon_{\square}=\left(\frac{a_{s}-a}{a}\right)$, sendo $a_{s}$ a constante de rede do substrato, no caso GaAs, e $a$ a constante de rede do PQ, InAs.
Portanto o potencial hidrostático, $V_{\text {hidro }}$ de acordo com a Ref. (RAICEVIC; TADIC, 2009) pode ser escrito da seguinte forma:

$$
V_{\text {hidro }}=a_{c}\left[\frac{C_{11}-C_{12}+6 C_{44}}{C_{11}+C_{12}+2 C_{44}}\right] \varepsilon_{\square}
$$

as constantes $C_{i j}$ são os módulos elásticos, que são determinados experimentalmente. $\mathrm{O}$ strain leva assim a um aumento da energia potencial da banda de condução.

O Hamiltoniano $\mathbf{k} \bullet \mathbf{p}$ tem uma simetria inerente que permite a separação do espaço de Hilbert em dois subespaços ortogonais (PRADO, et al., 2004). As funções de onda são expandidas em espinores de duas-componentes em cada subespaço de Hilbert, em termo das soluções exatas das componentes da diagonal do Hamiltoniano (1). Cada componente do espinor tem a seguinte forma (PRADO, 2013):

$$
\begin{aligned}
f_{n, s}^{m}(\rho, \theta, z)= & \frac{1}{\lambda}\left(\frac{\Gamma[n+m+1]}{2^{m+1} n !(\Gamma[m+1])^{2} \pi}\right)^{1 / 2} e^{-i m \theta} e^{-\frac{1}{4}\left(\frac{\rho}{\lambda}\right)^{2}}\left(\frac{\rho}{\lambda}\right)^{m} \\
& { }_{1} F_{1}\left(-n, m+1, \frac{1}{2}\left(\frac{\rho}{\lambda}\right)^{2}\right) \sqrt{\frac{1}{H_{z}}} \operatorname{sen}\left(\sin \left(\frac{1}{2}-\frac{z}{H_{z}}\right)\right),
\end{aligned}
$$

onde ${ }_{1} F_{1}$ é a função hipergeométrica confluente,

$\lambda=\sqrt{\frac{\hbar}{m^{*} \omega}}$ com $\omega=\sqrt{\omega_{0}^{2}+\omega_{c}^{2}}, \quad \omega_{c}=\frac{e B}{\underline{m}^{*}} \quad$ é a frequência ciclotrônica, $n=0,1,2 \ldots ; m \underline{\underline{m}}_{\ldots,-1,0}$, $1, \ldots$ e $s=1,2,3 \ldots$ Portanto as funções de onda, $\psi_{I(I I)}$, para cada subespaço I e II são escritas como,

$$
\left|\psi_{\mathrm{I}(\mathrm{II})}^{m}\right\rangle=\sum_{n, m, s}^{\infty}\left(\begin{array}{l}
C_{n, s}^{m(m-1)} f_{n, s}^{m(m-1)}|e \uparrow\rangle \\
C_{n, s}^{m+1(m)} f_{n, s}^{m+1(m)}|e \downarrow\rangle
\end{array}\right)
$$

sendoque $C_{n, s}^{m}$ sãoconstantes a serem determinadas. 


\section{Resultados}

Os parâmetros do InAs usados neste trabalho são, a massa efetiva do elétron $m e(\operatorname{InAs})=0,079 m^{*}$, onde $m *$ é a massa do elétron livre e os parâmetros relacionados com strain (VURGAFTMAN; MEYER, 2001): $\mathrm{C}_{11}=832,9 \mathrm{GPa}, \mathrm{C}_{12}=452,6 \mathrm{GPa}$, $\mathrm{C}_{44}=395,9 \mathrm{GPa}, \mathrm{C}_{3}=0,52 \mathrm{eV} . \mathrm{nm}, a_{c}=-5,08 \mathrm{eV}$. Os parâmetros de rede do GaAs e do InAs, são respectivamente, $a_{s}=5,66325 \AA$ e $a=6,0583 \AA$, portanto neste caso o strain é compressivo, pois $\Delta a$ $=a s-a<0$.

É útil introduzir o momento angular total $\mathbf{F}=\mathbf{J}+\mathbf{L}$, onde $\mathbf{J}$ é o momento angular total da função de Bloch, e $\mathbf{L}$ é o momento angular envelope. Como a projeção do momento angular total $F_{z}\left(j_{z}+m\right)$ é uma constante de movimento ela foi usada para indexar os níveis de energia em $\mathrm{B}=0 \mathrm{~T}$ da seguinte forma; nível $=\mathbf{0}: F_{\mathrm{z}}=1 / 2$ (spin= $) ; 1:-1 / 2(\downarrow) ; 2: 3 / 2(\uparrow) ; 3: 1 / 2(\downarrow) ; 4:-1 / 2(\uparrow)$; 5: $-3 / 2(\downarrow) ; 6: 5 / 2(\uparrow)$ e $7: 3 / 2(\downarrow)$. Os espectros de energia da banda de condução como função do campo magnético são mostrados na figura 1, $\operatorname{com} H_{z}$ $=20 \AA ̊$ e $E_{0}=7,5 \mathrm{meV}$ (que equivale a um raio $\rho \mathrm{de}$ $16 \AA ̊$, pois $\left.E_{0}=4 \hbar^{2} / m^{*} \rho^{2}\right)$. A fig. 1 a) é sem strain e sem ISO, a 1 b) é sem ISO e com strain, neste caso observa-se que aumentaram os valores das energias já que o strain comprime os elétrons no plano- $x y$. $\mathrm{Na} 1$ c) $\alpha$ é igual $20 \mathrm{meV} . n \mathrm{~m}$ e não se considera o strain, neste caso há quebra da degenerescência em campo magnético zero, assim como reordenamento dos níveis em campos baixos e repulsão em campos altos, como destacado na figura 2. Na fig. 1 d) estão incluídos a ISO, com $\alpha=20 \mathrm{meV} . n \mathrm{~m}$ e o strain.

Figura 1 - Níveis de energia dos elétrons na banda de condução para um PQ cilíndrico como função do campo magnético: a) sem strain e sem ISO, b) com strain e sem ISO, c) sem strain e com ISO e d) com ambos os efeitos. As linhas cheias representam os níveis pertencentes ao subespaço I e as pontilhadas ao subespaço II.

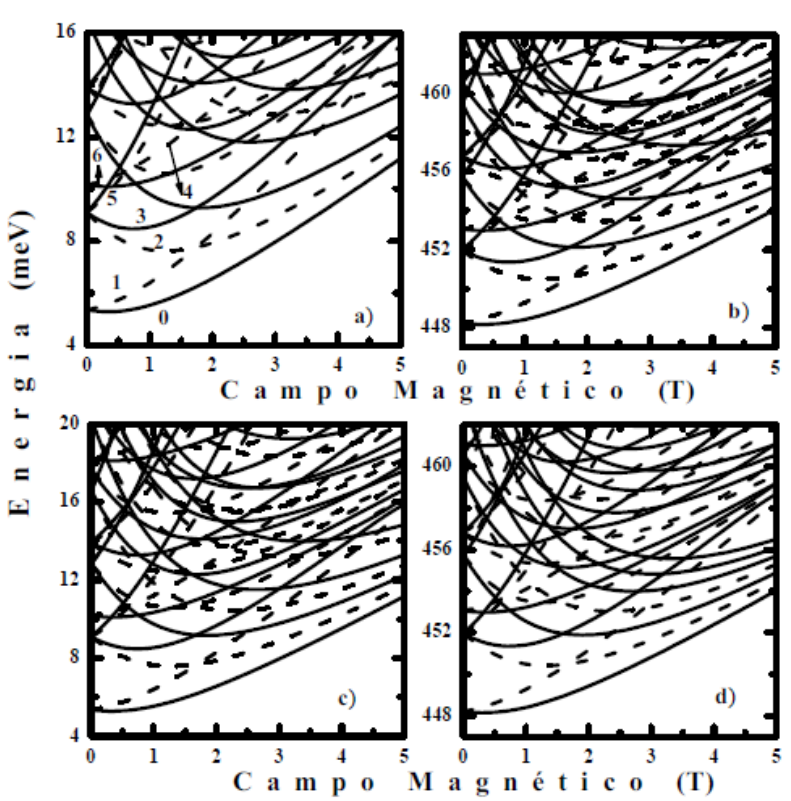

Fonte: $\mathrm{O}$ autor.

A figura 2 mostra uma ampliação dos níveis de energia da figura 1 , destacando os efeitos da ISO que em campo zero leva á quebra da degenerescência e a inversão dos níveis. Além disso, a ISO agindo sobre os níveis 4 e 7 leva a formação de um mini-gap de energia devido ao anti-cruzamento destes níveis em $\mathrm{B} \equiv 0,6 \mathrm{~T}$, o strain realça o mini-gap como pode ser visto na fig. 3 a). Este anti-cruzamento leva a uma troca do caráter de spin desses estados, ou seja, em campo nulo o nível 4 tem spin- $\uparrow$ e o nível 7 spin$\downarrow$, após o anti-cruzamento o nível 4 tem spin- $\downarrow$ e o nível 7 spin- $\uparrow$. A figura 3 b) mostra a variação do mini-gap em função de $\mathrm{E}_{0}$ para $\alpha$ fixo e três valores da meia-altura, onde se observa que o mini-gap deixa de existir, quando $\mathrm{E}_{0}$ é maior que $17,0 \mathrm{meV}$ para $H_{z}=15 \AA ̊$, 9,5meV para $H_{z}=20 \AA ̊$ e $7,5 \mathrm{meV}$, para $H_{z}=22 \AA ́$. Observa-se também que os valores do mini-gap passam por mínimos, os quais ocorrem devido aos acoplamentos entre os níveis de energia e como esses acoplamentos se modificam devido à complexa relação entre a razão $\rho / H_{z}$ (da energia de confinamento lateral $\mathrm{E}_{0} \sim 1 / \rho 2$, pela meia-altura do PQ), a 
Figura 2 - Ampliação da figura 1 destacando os efeitos da ISO e do strain em campos baixos: a) sem strain e ISSO, b) com strain, sem ISSO, as energias aumentaram, c) com ISSO e sem strain, separação dos níveis em $\mathrm{B}=0$ e reordenamento dos mesmos e d) ambos os efeitos incluídos, mostrando quebra da degenerescência em $\mathrm{B}=0$, reordenamento e aumento da energia.

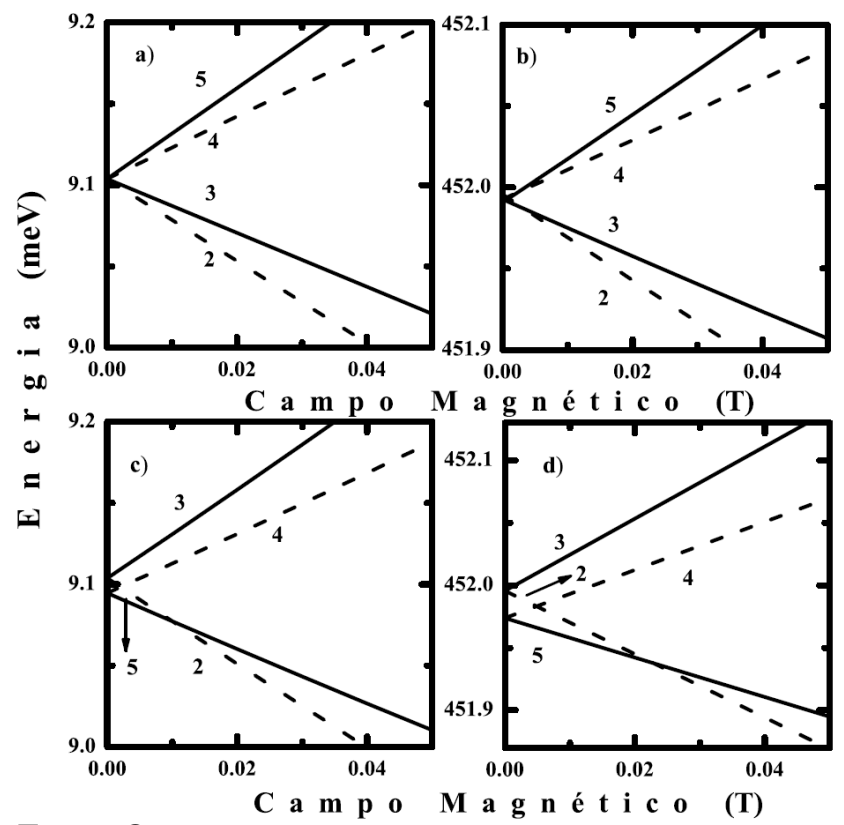

Fonte: $\mathrm{O}$ autor.

influência do strain, da ISO e do campo magnético. A figura 3 c) mostra a evolução do mini-gap como função da intensidade da ISO com e sem strain, mantidos $\mathrm{E}_{0}$ e $\mathrm{H}_{\mathrm{z}}$ fixos, novamente o efeito principal do strain é de aumentar a separação entre os níveis de energia. O campo magnético crítico, $\mathrm{B}_{\mathrm{c}}$, é o valor do campo onde ocorre o mini-gap, ele está plotado na figura $3 \mathrm{~d}$ ) como função de $\mathrm{E}_{0}$, para $\alpha$ fixo e três valores de $\mathrm{H}_{\mathrm{z}}$. Da figura observa-se que o $\mathrm{B}_{\mathrm{c}}$ diminui a medida que a energia de confinamento lateral aumenta, deixando de existir nos valores de $\mathrm{E}_{0}$ mencionados na discussão dos resultados da fig. 3 b). Por outro lado, para um dado valor de $\mathrm{E}_{0}$, diminuir $\mathrm{H}_{\mathrm{z}}$, aumenta os valores de $\mathrm{B}_{\mathrm{c}}$.
Figura 3 - a) Ampliação das figuras 1 a) à 1 d) destacando o mini-gap de energia. b) Variação do mini-gap de energia como função de $\mathrm{E}_{0}$. c) Variação do mini-gap como função de $\alpha$ e d) campo magnético crítico como função de $\mathrm{E}_{0}$.
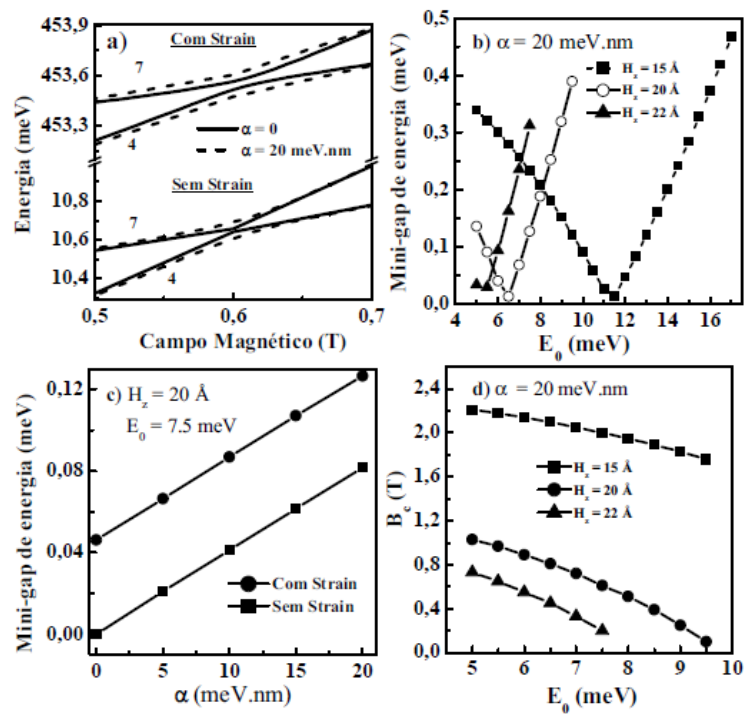

Fonte: $\mathrm{O}$ autor.

Para verificar as trocas de spin entre os níveis 4 e 7, calcula-se o valor esperado da projeção do spin na direção de quantização- $z$ em função do campo magnético, usando a seguinte expressão:

$$
\left\langle S_{z}\right\rangle=\left\langle\psi\left|S_{z}\right| \psi\right\rangle=\sum_{n, m, s=0}\left|C_{n, s, \uparrow}^{m}\right|^{2}-\left|C_{n, s, \downarrow}^{m}\right|^{2}
$$

Figura 4 - Valor esperado de $\left\langle S_{z}\right\rangle$ em unidades de $\hbar / 2$.

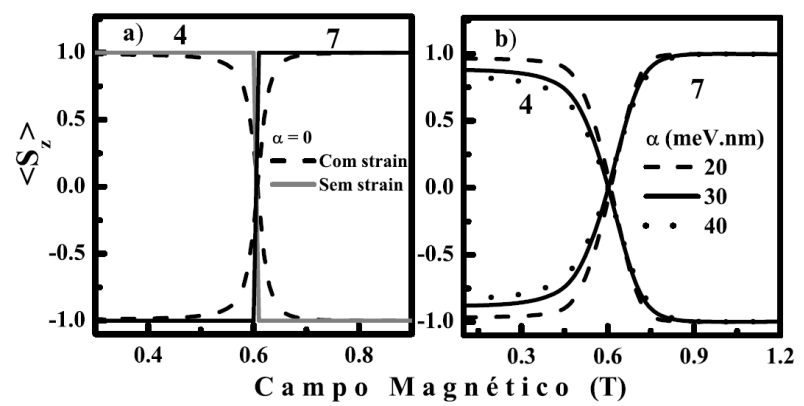

Fonte: $\mathrm{O}$ autor. 
Os resultados estão nas figuras 4 a) e 4 b)

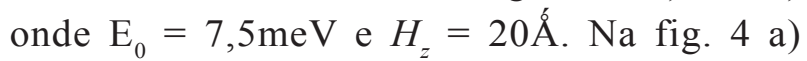
com a ISO desligada em $\mathrm{B}=0 \mathrm{~T}$ observa-se que o spin dominante do nível 4 é $\uparrow$ e do nível 7- $\downarrow$, tanto no caso com strain quanto no caso sem. À medida que o campo aumenta os referidos níveis trocam de spin de forma abrupta em $\mathrm{B}=0,6 \mathrm{~T}$, no caso sem strain e quando este é incluído ele comprime os níveis de energia modificando a interação entre eles de tal forma que a troca de spins ocorre de forma mais suave. Na fig. 4 b) a inclusão da ISO faz com que os estados deixem de ser puros em $\mathrm{B}=0 \mathrm{~T}$, ou seja a componente $\left\langle S_{z}\right\rangle$ não é igual a unidade, além disso quanto maior o $\alpha$ maior a mistura dos estados de spin e maior o valor dos mini-gaps de energia, tornando ainda mais suave a troca de spins.

A evolução do mini-gap como função de $\alpha$, por exemplo, pode ser estudada usando absorção óptica intrabanda entre o nível de energia fundamental da banda de condução e os níveis 4 e 7. Para isto usa-se luz incidente com polarização circular, cujas regras de seleção óptica permitem transições entre estados pertencentes a subespaços diferentes $(\mathrm{I} \rightarrow \mathrm{II}$ ou $\mathrm{II} \rightarrow \mathrm{I})$ e que conectam estados com $\Delta \mathrm{Fz}= \pm 1$ (LÓPEZ-RICHARD et al., 2005). Na fig. 5 têm-se os espectros de absorção, no caso em que a ISO é nula as transições permitidas para a luz incidente com polarização circular ocorre entre os níveis de energia $\mathbf{0} \uparrow \rightarrow \mathbf{4} \uparrow \mathrm{e}$ para $\sigma^{+}$entre os níveis $\mathbf{0} \uparrow \rightarrow \mathbf{2} \uparrow$, fig. 5 a). Quando a ISO não é nula para aparece a transição $0 \uparrow \rightarrow 7 \uparrow$, para $\mathrm{B}>0,6 \mathrm{~T}$, pois como discutido os estados 4 e 7 trocam o caráter de spin em aproximadamente $0,6 \mathrm{~T}$, resultando em um mini-gap de energia na absorção óptica, mostrado nas figuras 5 b), c) e d). À medida que a ISO aumenta, da figura 5 a) à 5 d) os acoplamentos entre os níveis de energia se tornam mais fortes aumentando o valor do mini-gap de energia.

Figura 5 - Espectros de absorção-óptica intrabanda para quatro valores de $\alpha, \mathrm{E}_{0}=7,5 \mathrm{meV}$ e $H_{z}=20 \AA ̊$.

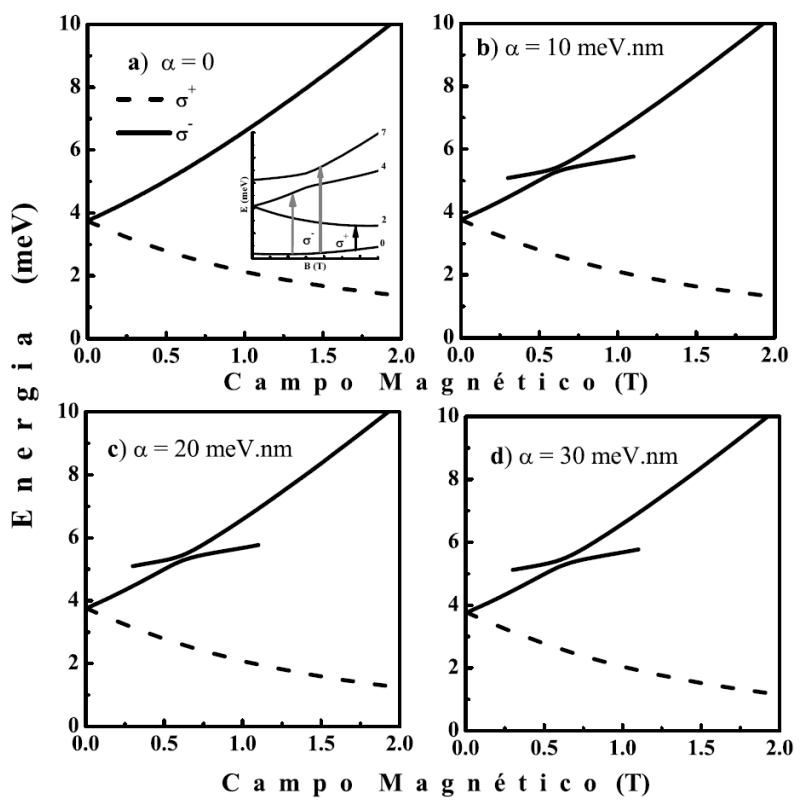

Fonte: $\mathrm{O}$ autor.

Como discutido na fig. $3 \mathrm{~d}$ ), para $H_{z}=20 \AA \hat{e} \mathrm{e}$ quando $\mathrm{E}_{0}=9,5 \mathrm{meV}$ não há troca de caráter do spin, então por exemplo, usando este valor da energia de confinamento lateral, pode-se manipular a troca de spins controlando a ISO como mostrado na fig. 6 . Finalmente, pode-se controlar as trocas de spin, o valor do mini-gap e as transições ópticas intrabanda através do ajuste de um dos parâmetros, $\alpha, H_{z}$ ou $\mathrm{E}_{0}$ mantendo os outros fixos de acordo com interesse em possíveis aplicações tecnológicas. 
Figura 6 - Valor esperado de $\left\langle S_{z}\right\rangle$ em unidades de $\hbar / 2$ para os elétrons confinados com $\mathrm{E}_{0}=9,5 \mathrm{meV}$.
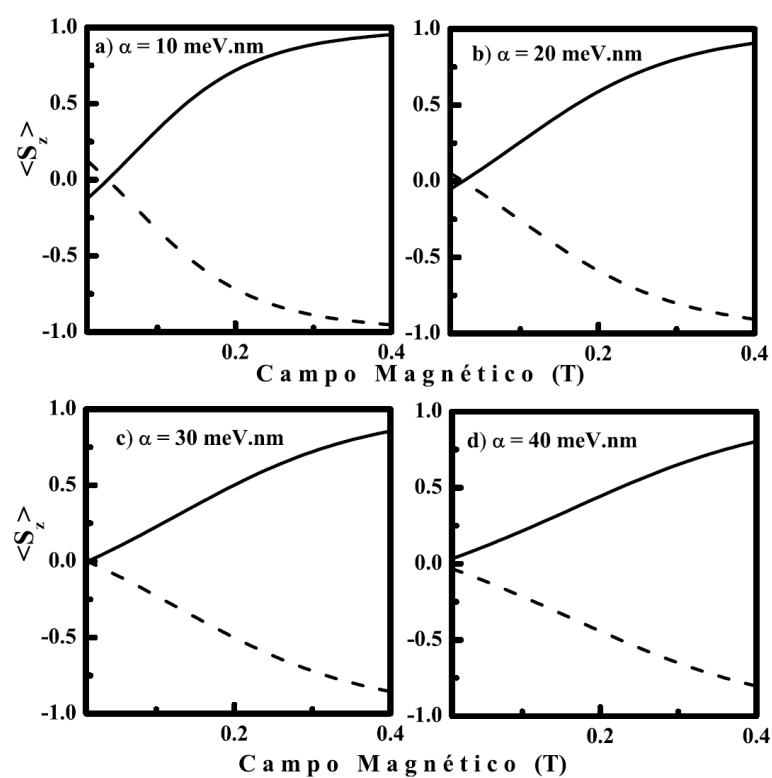

Fonte: $\mathrm{O}$ autor.

\section{Conclusões}

Neste trabalho apresentei um estudo sistemático sobre a influência da interação SO Rashba e do strain na banda de condução em um PQ cilíndrico de InAs com campo magnético externo. Os resultados mostram que a ISO age levantando a degenerescência mesmo em campo magnético nulo, assim como alterando a ordem dos níveis de energia excitados. Já o strain age comprimindo os níveis de energia realçando os efeitos da ISO, efeitos estes que podem ser observados através da absorção óptica intrabanda.

\section{Referências}

JHA, P. K.; KUMAR, M.; LAHON, S.; GUMBER, S.; MOHAN, M. Rashba spin orbit interaction effect on nonlinear optical properties of quantum dot with magnetic field. Superlattice and Microstructures, v. 65, p. 71-78, 2014.

KATO, I.; MYERS, R. C.; GOSSARD, A. C.; AWSCHALOM, D. D. Coherent spin manipulation without magnetic fields in strained semiconductors. Nature, v. 427, p. 50-53, 2004.
LI, Y; LI, Y.-Q. Strain-assisted spin manipulation in a quantum well. European Physical Journal B, v. 63, p. 493-500, 2008.

LÓPEZ-RICHARD, V.; ALCALDE, A. M.; PRADO, S. J.; MARQUES, G. E.; TRALLERO-GINER, C. Intraband magneto absorption as a probing for the quantum dot charge. Applied Physics Letters, v. 87, p. 231101-1-231101-3, 2005.

MANSELYAN, A.; CHAKRABORTY, T. Enhanced Rashba effect for holes in a quantum dot. Europhys. Lett., v. 88, p. 17003-1-17003-4, 2009.

NADJ-PERGE, S.; FROLOV, S. M.; BAKKERS, E. P. A. M.; KOUWENHOVEN, L. P. Spin-orbit qubit in a semiconductor nanowire. Nature, v. 468, p. 10841087, 2010.

PIETILÄINEN, P.; CHAKRABORTY, T. Energy levels and magneto-optical transitions in parabolic quantum dots with spin-orbit coupling. Physical Review B, v. 73, p. 155315-1-155315-17, 2006.

PRADO, S. J. Absorção-Óptica intrabanda em um ponto quântico parabólico com acoplamento spinórbita. Revista Ciências Exatas e Naturais, v. 15, n 1, p. 49-59, 2013.

PRADO, S. J.; TRALLERO-GINER, C.; ALCALDE, A. M.; LÓPEZ-RICHARD, V.; MARQUES, G. E. Magneto-optical properties of nanocrystals: Zeeman splitting, Physical Review B, v. 67, p. 165306-1165306-10, 2004.

RAICEVIC, N.; TADIC, M. Influence of strain on band Structure of Semiconductor nanostructures. Serbian Journal of Electrical Engineering, v. 6, n. 3, p. 461-469, 2009.

VURGAFTMAN, I.; MEYER, J. R. Band parameters for III-V compound semiconductors and their alloys. Journal of Applied Physics, v. 89, n. 11, p. 5815-5875, 2001.

Recebido em 7 Fevereiro 2015 - Received on February 7, 2015. Aceito em 7 Maio, 2015 - Accepted on May 7, 2015. 
